\title{
Upper Bound of Difference Operator on Some Matrix Domains
}

\author{
Lotfollah Karimi $^{1 *}$ and Maryam Sinaei ${ }^{2}$ \\ ${ }^{1}$ Department of Basic Science, Hamedan University of Technology, Hamedan, Iran \\ ${ }^{2}$ Department of mathematics, Azad university of Shiraz, Shiraz, branch, Shiraz, Iran \\ * Corresponding author
}

\section{Article Info}

Keywords: Cesàro matrix, Difference operator, Hilbert matrix, Norm, Sequence space.

2010 AMS: 26D15, 40C05, 40G05,

$47 B 37$.

Received: 18 November 2020

Accepted: 6 April 2021

Available online: 30 April 2021

\begin{abstract}
In this study, we investigate the norm of difference operator on some sequence spaces such as Hilbert and Cesàro matrix domains. Therefore the present study is a complement for those results obtained in [1].
\end{abstract}

\section{Introduction}

Let $p>1$ and $\omega$ denote the set of all real-valued sequences. The Banach space $\ell_{p}$ is the set of all real sequences $x=\left(x_{k}\right)_{k=0}^{\infty} \in \omega$ such that

$$
\|x\|_{\ell_{p}}=\left(\sum_{k=0}^{\infty}\left|x_{k}\right|^{p}\right)^{1 / p}<\infty .
$$

We use the notations $\Delta^{B}$ and $\Delta^{F}$ to indicate the backward and forward difference matrices, respectively. These matrices are defined by

$$
\delta_{j, k}^{B}=\left\{\begin{array}{cc}
1 & k=j \\
-1 & k=j-1 \\
0 & \text { otherwise, }
\end{array} \quad \text { and } \quad \delta_{j, k}^{F}=\left\{\begin{array}{cc}
1 & k=j \\
-1 & k=j+1 \\
0 & \text { otherwise. }
\end{array}\right.\right.
$$

Also Roopaei in [2] has introduced the notations $\ell_{p}\left(\Delta^{B}\right)$ and $\ell_{p}\left(\Delta^{F}\right)$ for the backward and forward difference sequence spaces defined by,

$$
\ell_{p}\left(\Delta^{B}\right)=\left\{x=\left(x_{n}\right): \sum_{n=1}^{\infty}\left|x_{n}-x_{n-1}\right|^{p}<\infty\right\}
$$

and

$$
\ell_{p}\left(\Delta^{F}\right)=\left\{x=\left(x_{n}\right): \sum_{n=1}^{\infty}\left|x_{n}-x_{n+1}\right|^{p}<\infty\right\},
$$

respectively. The domains $c_{0}\left(\Delta^{F}\right), c\left(\Delta^{F}\right)$ and $\ell_{\infty}\left(\Delta^{F}\right)$ of the forward difference matrix $\Delta^{F}$ in the spaces $c_{0}, c$ and $\ell_{\infty}$ are introduced by Kizmaz [3]. Aftermore, the domain $b v_{p}$ of the backward difference matrix $\Delta^{B}$ in the space $\ell_{p}$ have recently been investigated for $0<p<1$ by Altay and Başar [4], and for $1 \leq p \leq \infty$ by Başar and Altay [5].

The infinite Cesàro operator is defined by

$$
c_{j, k}= \begin{cases}\frac{1}{j+1} & 0 \leq k \leq j \\ 0 & \text { otherwise }\end{cases}
$$


for all $j, k \in \mathbb{N}$. That is,

$$
C=\left(\begin{array}{cccc}
1 & 0 & 0 & \cdots \\
1 / 2 & 1 / 2 & 0 & \cdots \\
1 / 3 & 1 / 3 & 1 / 3 & \cdots \\
\vdots & \vdots & \vdots & \ddots
\end{array}\right)
$$

This operator has the $\ell_{p}$-norm $\|C\|_{\ell_{p}}=p^{*}$, where $p^{*}$ is the conjugate of $p$ i.e. $\frac{1}{p}+\frac{1}{p^{*}}=1$.

Suppose that $N \geq 1$ is a real number. The generalized Cesàro matrix, $C^{N}=\left(c_{j, k}^{N}\right)$,

$$
c_{j, k}^{N}=\left\{\begin{array}{lc}
\frac{1}{j+N} & 0 \leq k \leq j \\
0 & \text { otherwise }
\end{array}\right.
$$

has the $\ell_{p}$-norm $\left\|C^{N}\right\|_{\ell_{p}}=p^{*}([6]$, Lemma 2.3), and the entries

$$
C^{N}=\left(\begin{array}{cccc}
\frac{1}{N} & 0 & 0 & \cdots \\
\frac{1}{1+N} & \frac{1}{1+N} & 0 & \cdots \\
\frac{1}{2+N} & \frac{1}{2+N} & \frac{1}{2+N} & \cdots \\
\vdots & \vdots & \vdots & \ddots
\end{array}\right)
$$

Note that, $C^{1}$ is the well-known Cesàro matrix $C$. For more examples

$$
C^{2}=\left(\begin{array}{cccc}
1 / 2 & 0 & 0 & \ldots \\
1 / 3 & 1 / 3 & 0 & \ldots \\
1 / 4 & 1 / 4 & 1 / 4 & \ldots \\
\vdots & \vdots & \vdots & \ddots
\end{array}\right) \quad \text { and } \quad C^{3}=\left(\begin{array}{cccc}
1 / 3 & 0 & 0 & \ldots \\
1 / 4 & 1 / 4 & 0 & \ldots \\
1 / 5 & 1 / 5 & 1 / 5 & \ldots \\
\vdots & \vdots & \vdots & \ddots
\end{array}\right)
$$

There are several research on the problem of finding the norm of operators on matrix domains while there are very limited papers about the norm of difference operators. Roopaei has recently computed the norm of backward difference operator on some sequence spaces and the present study is a complement for those results obtained in [1].

\section{Norm of operators on matrix domains}

The operator $T$ is called bounded, if the inequality $\|T x\|_{\ell_{p}} \leq K\|x\|_{\ell_{p}}$ holds for all sequences $x \in \ell_{p}$, while the constant $K$ is not depending on $x$. The constant $K$ is called an upper bound for operator $T$ and the smallest possible value of $K$ is called the norm of $T$.

The domain $X_{T}$ of an infinite matrix $T$ in a sequence space $X$ is defined as

$$
X_{T}=\{x \in \omega: T x \in X\}
$$

which is also a sequence space. It is easy to see that for an invertible matrix $T$, the matrix domain $T_{p}$ is a normed space with $\|x\|_{T_{p}}:=\|T x\|_{\ell_{p}}$. By using matrix domains of special triangular matrices in classical spaces, many authors have introduced and studied new Banach spaces. For the relevant literature, we refer to the papers $[7,8,9,10,11,12,13]$ and textbook [14]. Recently, Roopaei has computed the norm of operators on several matrix domains in $[2,15,16,17,18,19,20,21]$.

Lemma 2.1 ([18], Lemma 3.1). Let $U$ be a bounded operator on $\ell_{p}$ and $A_{p}$ and $B_{p}$ are two matrix domains such that $A_{p} \simeq \ell_{p}$.

- If BT is a bounded operator on $\ell_{p}$, then $T$ is a bounded operator from $\ell_{p}$ into $B_{p}$ and $\|T\|_{\ell_{p}, B_{p}}=\|B T\|_{\ell_{p}}$.

- If $T$ has a factorization of the form $T=U A$, then $T$ is a bounded operator from the matrix domain $A_{p}$ into $\ell_{p}$ and $\|T\|_{A_{p}, \ell_{p}}=\|U\|_{\ell_{p}}$.

- If $B T=U A$, then $T$ is a bounded operator from the matrix domain $A_{p}$ into $B_{p}$ and

$$
\|T\|_{A_{p}, B_{p}}=\|U\|_{\ell_{p}} .
$$

In particular, if $A T=U A$, then $T$ is a bounded operator from the matrix domain $A_{p}$ into $A_{p}$ and $\|T\|_{A_{p}}=\|U\|_{\ell_{p}}$. Also, if $T$ and A commute then $\|T\|_{A_{p}}=\|T\|_{\ell_{p}}$.

\subsection{Norm of difference operator on the Hilbert sequence space}

Recall the definition of Hilbert matrix $H=\left(h_{j, k}\right)$, which is defined by

$$
h_{j, k}=\frac{1}{j+k+1} \quad(j, k=0,1, \ldots) .
$$

That is

$$
H=\left(\begin{array}{cccc}
1 & 1 / 2 & 1 / 3 & \cdots \\
1 / 2 & 1 / 3 & 1 / 4 & \cdots \\
1 / 3 & 1 / 4 & 1 / 5 & \cdots \\
\vdots & \vdots & \vdots & \ddots
\end{array}\right)
$$


We know that $H$ is a bounded operator on $\ell_{p}$ with $\|H\|_{\ell_{p}}=\pi \csc (\pi / p)$ ([22], Theorem 323).

The sequence space associated with the Hilbert matrix, $H_{p}$, is defined by

$$
H_{p}=\left\{x=\left(x_{k}\right) \in \omega: \sum_{j=0}^{\infty}\left|\sum_{k=0}^{\infty} \frac{x_{k}}{j+k+1}\right|^{p}<\infty\right\},
$$

and has the following norm

$$
\|x\|_{H_{p}}=\left(\sum_{j=0}^{\infty}\left|\sum_{k=0}^{\infty} \frac{x_{k}}{j+k+1}\right|^{p}\right)^{\frac{1}{p}} .
$$

Theorem 2.2 ([22], Theorem 275). Let $p>1$ and $T=\left(t_{j, k}\right)$ be a matrix operator with $t_{j, k} \geq 0$ for all $j, k$. Suppose that $C, R$ are two strictly positive numbers such that

$$
\sum_{j=0}^{\infty} t_{j, k} \leq C \quad \text { for all } k, \quad \sum_{k=0}^{\infty} t_{j, k} \leq R \quad \text { for all } j
$$

bounds for column and row sums respectively. Then

$$
\|T\|_{\ell_{p}} \leq R^{1 / p^{*}} C^{1 / p} .
$$

The above theorem also known as Schur's theorem.

Theorem 2.3. The $\ell_{p}$ norm of the backward difference operator on the Hilbert matrix domain $H_{p}$, is the $\ell_{p}$-norm of forward difference operator on $H_{p}$ and

$(a)\left\|\Delta^{B}\right\|_{H_{p}, H_{p}}=\left\|\Delta^{F}\right\|_{\ell_{p}}$,

(b) $\left\|\Delta^{B}\right\|_{\ell_{p}, H_{p}} \leq 1$.

Proof. (a) Let $A=H \Delta^{B}$. The matrix $A=\left(a_{j, k}\right)$ has the entries

$$
a_{i, k}=\sum_{j=k, k+1} h_{i, j} \delta_{j, k}^{B}=\frac{1}{i+k+1}-\frac{1}{i+k+2}=\frac{1}{(i+k+1)(i+k+2)} .
$$

Obviously, $A$ is a symmetric matrix which implies that $H \Delta^{B}=\Delta^{F} H$. Now,

$$
\begin{aligned}
\left\|\Delta^{B}\right\|_{H_{p}, H_{p}} & =\sup _{x \in H_{p}} \frac{\left\|H \Delta^{B} x\right\|_{\ell_{p}}}{\|x\|_{H_{p}}}=\sup _{x \in H_{p}} \frac{\left\|\Delta^{F} H x\right\|_{\ell_{p}}}{\|H x\|_{\ell_{p}}} \\
& =\sup _{y \in \ell_{p}} \frac{\left\|\Delta^{F} y\right\|_{\ell_{p}}}{\|y\|_{\ell_{p}}}=\left\|\Delta^{F}\right\|_{\ell_{p}} .
\end{aligned}
$$

(b) Let $A$ be the matrix defined in part $(a)$. According to Lemma 2.1 part $(i)$

$$
\left\|\Delta^{B}\right\|_{\ell_{p}, H_{p}}=\left\|H \Delta^{B}\right\|_{\ell_{p}}=\|A\|_{\ell_{p}}
$$

By a simple calculation

$$
u_{k}=\sum_{j=0}^{\infty} a_{j, k}=\frac{1}{k+1}
$$

where $u_{k}$ is the $k^{\text {th }}$ column sum of $A$. Since $1=u_{0}>u_{1}>\cdots$ and $A$ is symmetric, hence $R$ and $C$ are both 1 in Schur's theorem. Therefore $\|A\|_{\ell_{p}} \leq 1$.

\subsection{Norm of difference operator on the Cesàro sequence space}

In this part of study, we intend to compute the norm of backward difference operator on the Cesàro sequence space. To do this we need the definition of the generalized Cesàro matrix domain.

The matrix domain associated with the generalized Cesàro matrix [15] is the set

$$
C_{p}^{N}=\left\{x=\left(x_{k}\right) \in \omega: \sum_{j=0}^{\infty}\left|\sum_{k=0}^{j} \frac{x_{k}}{j+N}\right|^{p}<\infty\right\},
$$

which has the following norm

$$
\|x\|_{C_{p}^{N}}=\left(\sum_{j=0}^{\infty}\left|\sum_{k=0}^{j} \frac{x_{k}}{j+N}\right|^{p}\right)^{\frac{1}{p}} .
$$

Note that, by letting $N=1$ we obtain the well-known Cesàro sequence space. 
Remark 2.4. In [23], $\mathrm{Ng}$ and Lee introduced the Cesàro sequence spaces $X_{p}$ and $X_{\infty}$ of non-absolute type as the domains of Cesàro matrix $C_{1}$ of order one in the spaces $\ell_{p}$ and $\ell_{\infty}$, where $1 \leq p<\infty$. Recently, Şengönül and Başar [24] studied the Cesàro sequence spaces $\widetilde{c}_{0}$ and $\widetilde{c}$ of non-absolute type as the domains of Cesàro matrix $C_{1}$ of order one in the spaces $c_{0}$ and c, also Roopaei et al [25] and Roopaei and Başar [10] have investigated the Cesáro space $C_{p}^{n}$ for $p \geq 1$ and $0<p<1$, respectively.

Theorem 2.5. The backward difference operator $\Delta^{B}$ is a bounded operator from $\ell_{p}$ into the generalized Cesàro matrix domain $C_{p}^{N}$ and

$$
\left\|\Delta^{B}\right\|_{\ell_{p}, C_{p}^{N}}=\frac{1}{N}
$$

In particular, the backward difference operator is a bounded operator from $\ell_{p}$ into $C_{p}$ and $\left\|\Delta^{B}\right\|_{\ell_{p}, C_{p}}=1$.

Proof. Let $D=C^{N} \Delta^{B}$. By a simple calculation, we deduce that the matrix $D=\left(d_{j, k}\right)$ is a diagonal matrix with entries

$$
D=\left(\begin{array}{cccc}
\frac{1}{N} & 0 & 0 & \cdots \\
0 & \frac{1}{1+N} & 0 & \cdots \\
0 & 0 & \frac{1}{2+N} & \cdots \\
\vdots & \vdots & \vdots & \ddots
\end{array}\right)
$$

Now, according to Lemma 2.1

$$
\left\|\Delta^{B}\right\|_{\ell_{p}, C_{p}^{N}}=\left\|C^{N} \Delta^{B}\right\|_{\ell_{p}}=\|D\|_{\ell_{p}}=\sup _{j} d_{j, j}=\frac{1}{N}
$$

In particular, for $N=1, C_{p}^{1}$ is the well-known Cesàro matrix domain $C_{p}$. Therefore we have the result.

Corollary 2.6. The generalized Copson operator is a bounded operator from $\ell_{p}$ into the forward difference matrix domain $\ell_{p}\left(\Delta^{F}\right)$ and

$$
\left\|C^{N t}\right\|_{\ell_{p}, \ell_{p}\left(\Delta^{F}\right)}=\frac{1}{N}
$$

In particular, Copson operator is a bounded operator from $\ell_{p}$ into $\ell_{p}\left(\Delta^{F}\right)$ and $\left\|C^{t}\right\|_{\ell_{p}, \ell_{p}\left(\Delta^{F}\right)}=1$.

Proof. According to Lemma 2.1 and previous theorem

$$
\begin{aligned}
\left\|C^{N t}\right\|_{\ell_{p}, \ell_{p}\left(\Delta^{F}\right)} & =\left\|\Delta^{F} C^{N t}\right\|_{\ell_{p}}=\left\|\left(C^{N} \Delta^{B}\right)^{t}\right\|_{\ell_{p}} \\
& =\left\|D^{t}\right\|_{\ell_{p}}=\sup _{j} d_{j, j}=\frac{1}{N},
\end{aligned}
$$

where $D$ is the diagonal matrix defined in the relation (2.1).

Theorem 2.7. The backward difference operator is a bounded operator from the generalized Copson space into the generalized Cesàro matrix domain and

$$
\left\|\Delta^{B}\right\|_{C_{p}^{N t}, C_{p}^{N}}=\left\|\Delta^{F}\right\|_{\ell_{p}}
$$

In particular, the backward difference operator is a bounded operator from the Copson matrix domain into the Cesàro matrix domain and $\left\|\Delta^{B}\right\|_{C_{p}^{t}, C_{p}}=\left\|\Delta^{F}\right\|_{\ell_{p}}$.

Proof. Through the proof of Theorem 2.5 we knew that $C^{N} \Delta^{B}=\Delta^{F} C^{N t}$. Now, according to Lemma 2.1 we have

$$
\begin{aligned}
\left\|\Delta^{B}\right\|_{C_{p}^{N t}, C_{p}^{N}} & =\sup _{x \in C_{p}^{N t}} \frac{\left\|\Delta^{B} x\right\|_{C_{p}^{N}}}{\|x\|_{C_{p}^{N t}}}=\sup _{x \in C_{p}^{N t}} \frac{\left\|C^{N} \Delta^{B} x\right\|_{\ell_{p}}}{\left\|C^{N t} x\right\|_{\ell_{p}}} \\
& =\sup _{x \in C_{p}^{N t}} \frac{\left\|\Delta^{F} C^{N t} x\right\|_{\ell_{p}}}{\left\|C^{N t} x\right\|_{\ell_{p}}}=\sup _{y \in \ell_{p}} \frac{\left\|\Delta^{F} y\right\|_{\ell_{p}}}{\|y\|_{\ell_{p}}} \\
& =\left\|\Delta^{F}\right\|_{\ell_{p}},
\end{aligned}
$$

that completes the proof.

Corollary 2.8. The generalized Copson operator is a bounded operator from the backward difference matrix domain $\ell_{p}\left(\Delta^{B}\right)$ into the forward difference space $\ell_{p}\left(\Delta^{F}\right)$ and

$$
\left\|C^{N t}\right\|_{\ell_{p}\left(\Delta^{B}\right), \ell_{p}\left(\Delta^{F}\right)}=p^{*}
$$

In particular, Copson operator is a bounded operator from $\ell_{p}\left(\Delta^{B}\right)$ into $\ell_{p}\left(\Delta^{F}\right)$ and $\left\|C^{t}\right\|_{\ell_{p}\left(\Delta^{B}\right), \ell_{p}\left(\Delta^{F}\right)}=p^{*}$.

Proof. The proof is similar to the proof of Theorem 2.7 . 
In sequel we intend to generalize the result of Theorem 2.7 for the backward difference operator of order $n$. At first we need some definitions. Let us recall the backward difference matrix of order $n, \Delta^{n}=\left(\delta_{j, k}^{n}\right)$, which is a lower triangular matrix with the entries

$$
\delta_{j, k}^{n}=\left\{\begin{array}{cl}
(-1)^{(j-k)}\left(\begin{array}{c}
n \\
j-k
\end{array}\right) & k \leq j \leq n+k \\
0 & \text { otherwise }
\end{array}\right.
$$

This matrix has the inverse $\Delta^{-n}=\left(\delta_{j, k}^{-n}\right)$ with the following entries

$$
\delta_{j, k}^{-n}=\left\{\begin{array}{cl}
\left(\begin{array}{cl}
n+j-k-1 \\
j-k
\end{array}\right) & j \geq k \\
0 & \text { otherwise }
\end{array}\right.
$$

Note that, for $n=1$, the backward difference of order 1 is $\Delta^{B}$ that was defined by relation (1.1).

The Hausdorff matrix $H^{\mu}=\left(h_{j, k}\right)_{j, k=0}^{\infty}$, is defined by:

$$
h_{j, k}= \begin{cases}\int_{0}^{1}\left(\begin{array}{l}
j \\
k
\end{array}\right) \theta^{k}(1-\theta)^{j-k} d \mu(\theta) & 0 \leq k \leq j \\
0 & k>j,\end{cases}
$$

where $\mu$ is a probability measure on $[0,1]$. The Hausdorff matrix contains some famous classes of matrices. By letting $d \mu(\theta)=$ $n(1-\theta)^{n-1} d \theta$ in the definition of the Hausdorff matrix, the Cesàro matrix of order $n, C^{n}=\left(c_{j, k}^{n}\right)$, is defined as follows

$$
c_{j, k}^{n}= \begin{cases}\frac{\left(\begin{array}{c}
n+j-k-1 \\
j-k
\end{array}\right)}{\left(\begin{array}{c}
n+j \\
j
\end{array}\right)} & 0 \leq k \leq j \\
0 & \text { otherwise }\end{cases}
$$

Note that, $C^{1}$ is the well-known Cesàro matrix $C$.

The sequence space $C_{p}^{n}$ is defined as the set of all sequences whose $C^{n}$-transforms are in the space $\ell_{p}$; that is

$$
C_{p}^{n}=\left\{x=\left(x_{j}\right) \in \omega: \sum_{j=0}^{\infty}\left|\frac{1}{\left(\begin{array}{c}
n+j \\
j
\end{array}\right)} \sum_{k=0}^{j}\left(\begin{array}{c}
n+j-k-1 \\
j-k
\end{array}\right) x_{k}\right|^{p}<\infty\right\}
$$

which is a Banach space with the norm

$$
\|x\|_{C_{p}^{n}}=\left(\sum_{j=0}^{\infty}\left|\frac{1}{\left(\begin{array}{c}
n+j \\
j
\end{array}\right)} \sum_{k=0}^{j}\left(\begin{array}{c}
n+j-k-1 \\
j-k
\end{array}\right) x_{k}\right|^{p}\right)^{1 / p} .
$$

The Copson matrix domain $C_{p}^{n t}$ is defined similarly which is isomorphic to the $\ell_{p}$ space by Theorem 2.3 of [18]. Roopaei in [17], through the proof of Corollary 3.6, has showed that $C^{n} \Delta^{n_{B}}$ is a diagonal matrix. Hence $C^{n} \Delta^{n_{B}}=\Delta^{n_{F}} C^{n t}$, where $\Delta^{n_{F}}$ is the forward difference operator of order $n$.

Now, as a result of Lemma 2.1 part (iii), we have the following result.

Theorem 2.9. The backward difference operator of order $n, \Delta^{n_{B}}$, is a bounded operator from the Copson matrix domain into the Cesàro matrix domain and

$$
\left\|\Delta^{n_{B}}\right\|_{C_{p}^{n t}, C_{p}^{n}}=\left\|\Delta^{n_{F}}\right\|_{\ell_{p}} .
$$

In particular, the backward difference operator is a bounded operator from the Copson matrix domain into the Cesàro matrix domain and $\left\|\Delta^{B}\right\|_{C_{p}^{t}, C_{p}}=\left\|\Delta^{F}\right\|_{\ell_{p}}$.

We have also the following corollary which has a proof similar to the above theorem.

Corollary 2.10 ([18], Theorem 4.3). The Copson matrix of order $n, C^{n}$, is a bounded operator from $\ell_{p}\left(\Delta^{n_{B}}\right)$ into $\ell_{p}\left(\Delta^{n_{F}}\right)$ and

$$
\left\|C^{n}\right\|_{\ell_{p}\left(\Delta^{n_{B}}\right), \ell_{p}\left(\Delta^{n_{F}}\right)}=\frac{\Gamma(n+1) \Gamma\left(1 / p^{*}\right)}{\Gamma\left(n+1 / p^{*}\right)} .
$$

In particular, the Copson matrix is a bounded operator from $\ell_{p}\left(\Delta^{B}\right)$ into $\ell_{p}\left(\Delta^{F}\right)$ and

$$
\|C\|_{\ell_{p}\left(\Delta^{B}\right), \ell_{p}\left(\Delta^{F}\right)}=p^{*}
$$

Theorem 2.11. Let $n, s$ and $m$ are non-negative integers that $n=s+m$. The backward difference operator of order $n, \Delta^{n_{B}}$, is a bounded operator from the matrix domain $\ell_{p}\left(\Delta^{m_{B}}\right)$ into the Cesàro matrix domain $C_{p}^{s}$ and

$$
\left\|\Delta^{n_{B}}\right\|_{\ell_{p}\left(\Delta^{\left.m_{B}\right), C_{p}^{s}}\right.}=1 .
$$


Proof. From the relation (2.2), one can see that the Cesàro matrix of order $n$ and its inverse can be rewritten based on the backward difference operator and of order $-n$ and its inverse. For $j \geq k$, we have

$$
c_{j, k}^{n}=\frac{\left(\begin{array}{c}
n+j-k-1 \\
j-k
\end{array}\right)}{\left(\begin{array}{c}
n+j \\
j
\end{array}\right)}=\frac{\delta_{j, k}^{-n}}{\left(\begin{array}{c}
n+j \\
j
\end{array}\right)}, \quad \text { and } \quad c_{j, k}^{-n}=\delta_{j, k}^{n}\left(\begin{array}{c}
n+k \\
k
\end{array}\right) .
$$

Let us first compute the matrix $C^{s} \Delta^{n_{B}}$.

$$
\left(C^{s} \Delta^{n_{B}}\right)_{j, k}=\sum_{i} \frac{\Delta_{j, i}^{-s_{B}} \Delta_{j, k}^{n_{B}}}{\left(\begin{array}{c}
s+j \\
j
\end{array}\right)}=\frac{1}{\left(\begin{array}{c}
s+j \\
j
\end{array}\right)} \Delta_{j, k}^{m}
$$

Hence, $C^{s} \Delta^{n_{B}}=U \Delta^{m_{B}}$, where $U=\left(u_{j, k}\right)$ is the diagonal matrix defined as $u_{j, j}=\frac{1}{\left(\begin{array}{c}s+j \\ j\end{array}\right)}$. Now, according to the Lemma 2.1 we have

$$
\begin{aligned}
\left\|\Delta^{n_{B}}\right\|_{\ell_{p}\left(\Delta^{m_{B}}\right), C_{p}^{s}} & =\sup _{x \in \ell_{p}\left(\Delta^{m_{B}}\right)} \frac{\left\|\Delta^{n_{B}} x\right\|_{C_{p}^{s}}}{\|x\|_{\ell_{p}\left(\Delta^{m_{B}}\right)}}=\sup _{x \in \ell_{p}\left(\Delta^{m_{B}}\right)} \frac{\left\|C^{s} \Delta^{n_{B}} x\right\|_{\ell_{p}}}{\left\|\Delta^{m_{B}} x\right\|_{\ell_{p}}} \\
& =\sup _{x \in \ell_{p}\left(\Delta^{m_{B}}\right)} \frac{\left\|U \Delta^{m_{B}} x\right\|_{\ell_{p}}}{\left\|\Delta^{m_{B}} x\right\|_{\ell_{p}}}=\sup _{y \in \ell_{p}} \frac{\|U y\|_{\ell_{p}}}{\|y\|_{\ell_{p}}} \\
& =\|U\|_{\ell_{p}}=\sup _{j} u_{j, j}=1 .
\end{aligned}
$$

Corollary 2.12. Let $n, s$ and $m$ are non-negative integers that $n=s+m$. The backward difference operator of order $n, \Delta^{n_{B}}$, is a bounded operator from the matrix domain $\ell_{p}\left(\Delta^{m_{B}}\right)$ into the matrix domain $\ell_{p}\left(\Delta^{s_{B}}\right)$ and

$$
\left\|\Delta^{n_{B}}\right\|_{\ell_{p}\left(\Delta^{m_{B}}\right), \ell_{p}\left(\Delta^{s} B\right)}=1
$$

Proof. The proof is similar to the proof of the above theorem.

\section{Acknowledgement}

The author wish to thank the referee for a careful reading and valuable comments on the original draft.

\section{References}

[1] H. Roopaei, D. Foroutannia, The norm of backward difference operator $\Delta^{n}$ on certain sequence spaces, Oper. Matrices, 12(3) (2018), 867-880.

[2] H. Roopaei, Norm of Hilbert operator on sequence spaces, J. Inequal. Appl., 2020(117), (2020).

[3] H. Kizmaz, On certain sequence spaces I, Canad. Math. Bull., 25(2) (1981), 169-176.

[4] B. Altay, F. Başar, The fine spectrum and the matrix domain of the difference operator $\Delta$ on the sequence space $\ell_{p}$, $(0<p<1)$, Commun. Math. Anal., 2(2) (2007), 1-11.

[5] F. Basar, B. Altay, On the space of sequences of p-bounded variation and related matrix mappings, Ukrainian Math. J., 55(1) (2003), 136-147.

[6] C. P. Chen, D. C. Luor, Z. y. Ou, Extensions of Hardy inequality, J. Math. Anal. Appl., 273 (2002), 160-171.

[7] B. Altay, F. Başar, Certain topological properties and duals of the matrix domain of a triangle matrix in a sequence space, J. Math. Anal. Appl., 336(1) (2007), 632-645.

[8] E. E. Kara, M. İlkhan, Some properties of generalized Fibonacci sequence spaces, Linear Multilinear Algebra, 64(11) (2016), $2208-2223$.

[9] F. Başar, Domain of the composition of some triangles in the space of p-summable sequences, AIP Conference Proceedings, 1611 (2014), $348-356$.

[10] H. Roopaei, F Başar, On the spaces of Cesàro absolutely p-summable, null, and convergent sequences, Math. Methods Appl. Sci., 44(5) (2021), 3670-3685.

[11] H. Roopaei, T. Yaying, Quasi-Cesàro matrix and associated sequence spaces, Turk. J. Math., 45(1) (2021), 153-166.

[12] H. Roopaei, M. İlkhan, Fractional Cesàro matrix and its associated sequence space, Concr. Oper., 8(1), (2021), 24-39.

[13] M. İlkhan, E. E. Kara, A new Banach space defined by Euler totient matrix operator, Oper. Matrices, 13(2) (2019), 527-544.

[14] F. Başar, Summability Theory and Its Applications, Bentham Science Publishers, İstanbul, 2012.

[15] H. Roopaei, D. Foroutannia, The norm of matrix operators on Cesàro weighted sequence space, Linear Multilinear Algebra, 67(1) (2019), $175-185$.

[16] H. Roopaei, D. Foroutannia, The norms of certain matrix operators from $\ell_{p}$ spaces into $\ell_{p}\left(\Delta^{n}\right)$ spaces, Linear Multilinear Algebra, 67(4) (2019), 767-776.

[17] H. Roopaei, Norms of summability and Hausdorff mean matrices on difference sequence spaces, Math. Inequal. Appl., 22(3) (2019), 983-987.

[18] H. Roopaei, A study on Copson operator and its associated sequence spaces, J. Inequal. Appl., 2020(120) (2020).

[19] H. Roopaei, A study on Copson operator and its associated sequence spaces II, J. Inequal. Appl., 2020(239) (2020).

[20] H. Roopaei, Bounds of operators on the Hilbert sequence space, Concr. Oper., 7 (2020), 155-165.

[21] H. Roopaei, Binomial operator as a Hausdorff operator of the Euler type, Constr. Math. Anal., 3(4) (2020), 165-177.

[22] G. H. Hardy, J. E. Littlewood, G. Polya, Inequalities, 2nd edition, Cambridge University Press, Cambridge, 2001.

[23] Ng P-N, Lee P-Y, Cesàro sequence spaces of non-absolute type, Comment. Math. Prace Mat., 20(2) (1978), 429-433.

[24] M. Şengönül, F. Başar, Cesàro sequence spaces of non-absolute type which include the spaces $c_{0}$ and c, Soochow J. Math., $31(1)$ (2005), $107-119$.

[25] H. Roopaei, D. Foroutannia, M. İlkhan, E. E. Kara, Cesàro Spaces and Norm of Operators on These Matrix Domains, Mediterr. J. Math., 17, 121 (2020).

[26] G. Bennett, Factorizing the classical inequalities, Mem. Amer. Math. Soc., 576 (1996). 